\title{
Modelagem Matemática de Propulsores Eletromecânicos utilizando os Estimadores Gauss-Newton e Levenberg-Marquardt
}

\author{
Caroline Luft ${ }^{1}$ \\ Manuel Martín Pérez Reibold ${ }^{2}$ \\ Dionatan Breskovit de Matos $^{3}$ \\ Eduardo Post 4 \\ Ivan Paulo Canal ${ }^{5}$ \\ Nelize Fracaro ${ }^{6}$ \\ Programa de Pós-Graduação em Modelagem Matemática, UNIJUÍ
}

\begin{abstract}
Resumo. As aeronaves do tipo multirrotor têm sido crescentemente investigadas, por suas vantagens como percepção tridimensional, mobilidade, estabilidade de voo e baixo custo de construção quando comparadas a outras aeronaves. Isto desafia a área de controle e requer a aquisição do modelo matemático do conjunto de propulsão eletromecânico. Para encontrar este modelo, utilizou-se de características específicas dos parâmetros do estimador associados à técnica de modelagem caixa preta. Neste estudo, demonstra-se o sistema propulsor eletromecânico, seu funcionamento e o modelo obtido com os estimadores de Gauss-Newton e Levenger-Marquardt, onde observou-se que o modelo utilizando o método de Gauss-Newton apresentou melhor resultado.
\end{abstract}

Palavras-chave. Identificação de Sistemas, Caixa Preta, ARMAX, VANTs, Quadricóptero

\section{Introdução}

A teoria de controle de sistemas visa incorporar suas características no tratamento de sistemas com incertezas. O controle adaptativo tem a capacidade de ajustar o sistema ao longo do tempo em resposta às mudanças adquiridas nos parâmetros do mesmo, sendo assim, uma motivação no desenvolvimento de aeronaves não tripuladas.

Os controladores adaptativos possuem um mecanismo de estimação de parâmetros, cuja finalidade é minimizar o erro entre a saída do sistema a controlar e a saída de um modelo de referência. No controle direto a estimação está implícita, o que propicia a adaptação por

\footnotetext{
${ }^{1}$ carol luft@hotmail.com

${ }^{2}$ manolo@unijui.edu.br

${ }^{3}$ brekovit.mat@gmail.com

${ }^{4}$ edupostmat@gmail.com

${ }^{5}$ ivan.canal@iffarroupilha.edu.br

${ }^{6}$ nelize_fracaro@hotmail.com
} 
desempenho. Enquanto no controle indireto, a estimação é explícita e propicia a adaptação por parâmetros.

Durante o voo de aeronaves nem todos os fenômenos físicos que ocorrem, principalmente nos propulsores, são passíveis de observação. Dessa forma, os modelos matemáticos dos propulsores são pouco consistentes, podendo ocasionar falhas ao controlar a aeronave, $[3,4]$. Nesse contexto, a identificação de sistemas é um processo que permite estimar parâmetros e verificar o desempenho comportamental de sistemas.

Nesse contexto, este trabalho se propõe obter o modelo matemático ARMAX (AutoRegressive Moving Average with Exogenous inputs) que descreve o desempenho comportamental de propulsores eletromecânicos. A determinação dos parâmetros característicos obter-se-á pela utilização dos estimadores de Gauss-Newton e de Levenberg-Marquardt. A diretriz metodológica consiste na realização das etapas da Identificação de Sistemas. A validação do modelo e seus parâmetros se dão por comparação entre os dados do sistema real e o modelo matemático obtido. O resultado desta investigação é uma contribuição à comunidade científica que modela matematicamente VANTs (Veículos Aéreos Não Tripulados).

O texto deste trabalho está organizado da seguinte forma: na seção 2 apresenta-se o princípio de funcionamento dos propulsores eletromecânicos e modelo matemático a ser utilizado. A seção 3 contém a metodologia, a qual se desenvolve a partir das etapas da Identificação de Sistemas. Na seção 4 são apresentados os resultados e sua discussão. Por fim, a seção 5 traz as conclusões e trabalhos futuros.

\section{Modelagem Matemática de Propulsores Eletromecânicos}

\subsection{Propulsores eletromecânicos}

O sistema de propulsão eletromecânico é constitúido por uma hélice, por um motor Brushless e por um ESC (Electronic Speed Control). Estes componentes funcionam da seguinte forma: o gerador de PWM (Pulse Width Modulation) aplica um sinal de tensão no ESC. Este ao receber o sinal, o divide em três outros sinais, normalmente trapezoidais e defasados entre si em $120^{\circ}$. Esses sinais amplificados energizam os enrolamentos do motor brushless de forma comutativa gerando um campo eletromagnético. Através do campo eletromagnético entre o enrolamento energizado e os imãs permanentes ocorre a rotação do motor. Ao eixo do rotor está conectada uma hélice que ao girar desloca a aeronave. A energia para este funcionamento é fornecida por uma bateria.

\subsection{Modelo matemático ARMAX}

A representação matemática utilizada é o ARMAX (Auto-Regressive Moving Average with exogenous inputs) por apresentar mais flexibilidade no manuseio de modelos que contém distúrbios. Esta representação é apresentada pela seguinte estrutura polinomial, [2]:

$$
y(k)=\frac{q^{-d} B\left(q^{-1}\right)}{A\left(q^{-1}\right)} u(k-1)+\frac{C\left(q^{-1}\right)}{A\left(q^{-1}\right) D\left(q^{-1}\right)} e(k)
$$


onde: $A(q), B(q), C(q)$ são matrizes polinomiais, $u(k)$ é o sinal de entrada, $q-1$ é o operador de atraso da entrada tal que $u(k) q-1=u(k-1), y(k)$ é o sinal de saída, $e(k)$ é o sinal de perturbação e $d$ é o retardo ou tempo morto, em múltiplos do período de amostragem $(d \geq 0)$. Os polinômios são definidos por:

$$
\begin{aligned}
& A(q)=1+a_{1} q^{-1}+a_{2} q^{-2}+\cdots+a_{n_{a}} q^{n_{a}} \\
& B(q)=b_{0}+b_{1} q^{-1}+b_{2} q^{-2}+\cdots+b_{n_{b}} q^{n_{b}} \\
& C(q)=1+c_{1} q^{-1}+c_{2} q^{-2}+\cdots+c_{n_{c}} q^{n_{c}} \\
& D(q)=1+d_{1} q^{-1}+d_{2} q^{-2}+\cdots+d_{n_{d}} q^{n_{d}}
\end{aligned}
$$

sendo, $n_{a}, n_{b}, n_{c}$ e $n_{d}$ os graus dos polinômios $A(q), B(q), C(q), D(q)$ em função do operador de atraso $q$, respectivamente.

\subsection{Estimador de Gauss-Newton}

Este estimador é uma modificação do método de Newton e é utilizado na resolução de problemas de mínimos quadrados não lineares. Apesar de ser usado apenas para minimizar uma soma de valores de função quadrada, tem a vantagem de que não são necessárias as derivadas segundas, que podem ser desafiadoras para calcular [1].

\subsection{Estimador Levenberg-Marquardt}

Destina-se a resolver o problema causado pelo fato da matriz $A_{k}^{T} A_{k}$, do sistema do método de Gauss-Newton ser singular. Pode ter convergência local lenta em problemas de grandes resíduos ou acentuadamente não lineares [1].

\section{Metodologia}

A pesquisa realizada neste trabalho investigativo é aplicada, quantitativa, exploratória e experimental. Utiliza-se do método dedutivo, da observação sistemática e da realização de testes como técnica. As variáveis discretas envolvidas no estudo são a corrente $i(k)$ e a velocidade de rotação do motor $\omega(k)$ do sistema de propulsão, ambas ilustradas na Figura 1, (a) e (b) respectivamente. O tempo de amostragem obedece ao critério do teorema de Shannon/Nyquist [1], o qual estabelece que a frequência de amostragem seja o dobro da maior frequência do sinal amostrado. O valor utilizado para o intervalo de amostragem é $0,04 \mathrm{~s}$.

A coleta de dados é realizada por meio da plataforma experimental para propulsores eletromecânicos desenvolvida nos laboratórios do Curso de Engenharia Elétrica da UNIJúI. Está constitúida por uma gangorra e um console de controle conforme ilustrado na Figura 2. O propulsor eletromecânico ensaiado é constitúido de um motor brushless, marca Turnigy, modelo 2826, $1400 \mathrm{kV}$; uma hélice 9 x 3,800, um ESC marca Redbrick, 24 A, e uma bateria Lítio Íon de $11 \mathrm{~V}, 1200 \mathrm{mAh}$.

A partir da coleta de dados, a metodologia aplicada consiste em aplicar os testes de estacionariedade, ADF e KPSS, de forma a garantir que o modelo obtido seja não espúrio. 


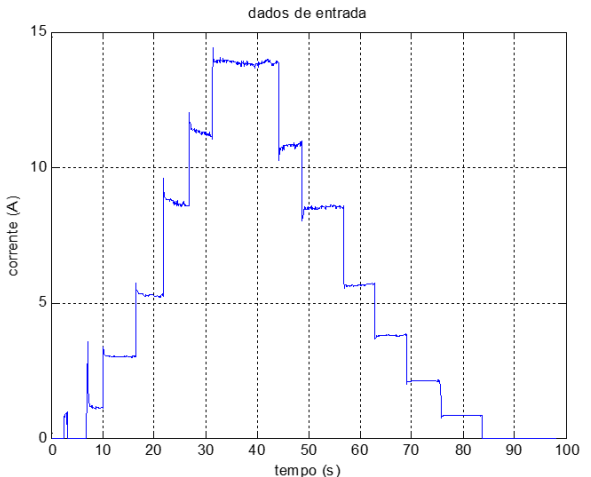

(a) Corrente $i(k)$

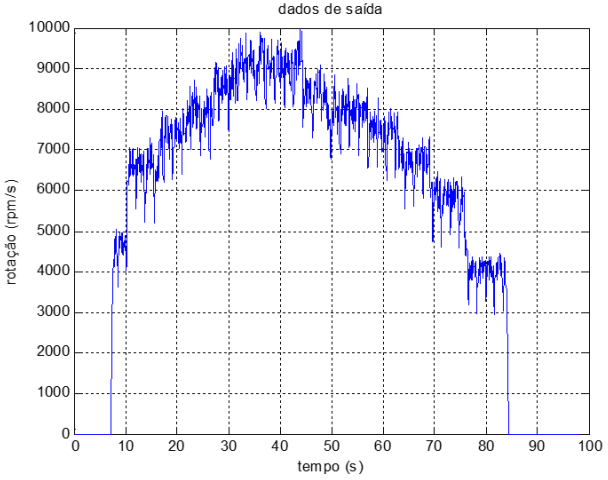

(b) Velocidade angular $\omega(k)$.

Figura 1: Dados coletados.

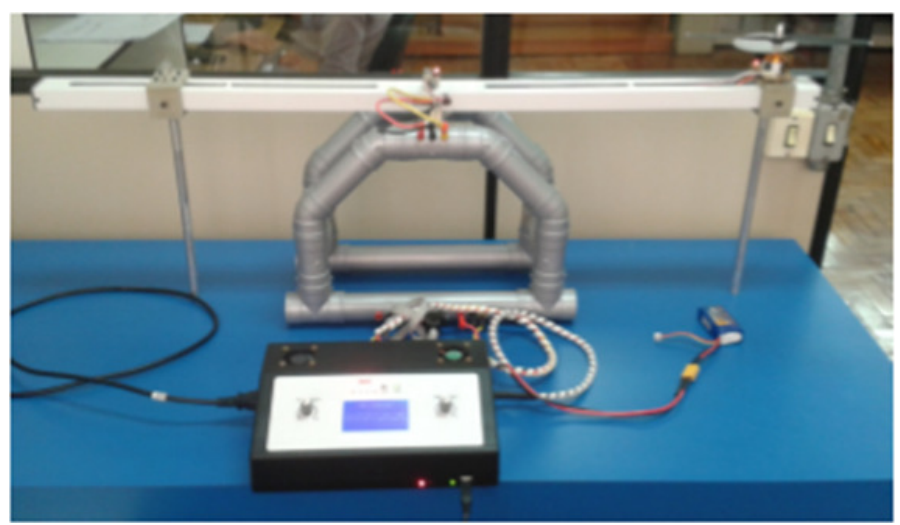

Figura 2: Plataforma experimental para propulsores eletromecânicos.

Em seguida, é feita a identificação da estrutura do modelo por meio das funções de autocorrelação (ACF) e autocorrelação parcial (PACF). Para a estimação de parâmetros utiliza-se os métodos de Gauss-Newton e Levenberg-Marquardt por serem estimadores próprios para modelo ARMAX. Por fim, pela comparação da simulação do modelo com os dados da plataforma e análise residual, o modelo é validado. Cabe ressaltar que todos os testes aplicados para a obtenção do modelo, são realizados a partir de funções implementadas no programa MatLab.

\section{Resultados e Discussoẽs}

A estrutura do modelo é da forma (p,q,r) onde $p$ representa o número de atrasos do processo (AR) obtido pela PACF (função de autocorrelação parcial) do sinal de saída, $q$ representa o número de atrasos do processo (MA) obtido pela ACF (função de autocorrelação) do sinal de saída, e $r$ representa o número de atrasos da série de entrada do modelo. Desta forma, por meio da comparação entre as características observadas na ACF e PACF, 
o modelo proposto é um modelo ARMAX de ordem $(7,5,4)$.

Após a escolha da representação e da estrutura do modelo, estimam-se os valores dos coeficientes de cada polinômio que compõe o modelo de acordo com cada método. As Tabelas 1 e 2 apresentam os parâmetros identificados através dos métodos de Gauss-Newton e Levenberg-Marquardt.

Tabela 1: Parâmetros do modelo ARMAX $(7,5,4)$ pelo método de Gauss-Newton.

\begin{tabular}{|c|c|c|c|c|c|c|c|c|}
\hline & 1 & $q^{-1}$ & $q^{-2}$ & $q^{-3}$ & $q^{-4}$ & $q^{-5}$ & $q^{-6}$ & $q^{-7}$ \\
\hline $\mathrm{A}(\mathrm{q})$ & 1,0000 & $-1,3350$ & $-1,1740$ & 2,2090 & $-0,6449$ & $-0,4176$ & 0,8192 & $-0,4557$ \\
\hline $\mathrm{B}(\mathrm{q})$ & 83,3400 & $-123,400$ & $-43,10$ & 123,20 & $-40,000$ & & & \\
\hline $\mathrm{C}(\mathrm{q})$ & 1,000 & $-0,5115$ & $-0,9415$ & 0,5280 & $-0,04192$ & & & \\
\hline
\end{tabular}

Tabela 2: Parâmetros do modelo ARMAX $(7,5,4)$ pelo método de Levenberg-Marquardt.

\begin{tabular}{|c|c|c|c|c|c|c|c|c|}
\hline & 1 & $q^{-1}$ & $q^{-2}$ & $q^{-3}$ & $q^{-4}$ & $q^{-5}$ & $q^{-6}$ & $q^{-7}$ \\
\hline $\mathrm{A}(\mathrm{q})$ & 1,0000 & $-0,8966$ & $-0,5231$ & $-0,1798$ & 0,1235 & 0,9714 & $-0,5923$ & 0,09691 \\
\hline $\mathrm{B}(\mathrm{q})$ & 280,30 & $-13,790$ & $-469,00$ & $-141,00$ & 343,50 & & & \\
\hline $\mathrm{C}(\mathrm{q})$ & 1,0000 & 0,2131 & $-0,3890$ & $-0,5214$ & $-0,3027$ & & & \\
\hline
\end{tabular}

As Tabelas 1 e 2 exibem os coeficientes dos polinômios $A(q), B(q)$ e $C(q)$ em função do operador de atraso q. Na linha do polinômio $A(q)$ são os valores correspondentes aos coeficientes da variável de saída do sistema; na linha $B(q)$ são os valores dos coeficientes da variável de entrada e na linha $C(q)$ são os valores correspondentes do processo rúido do modelo.

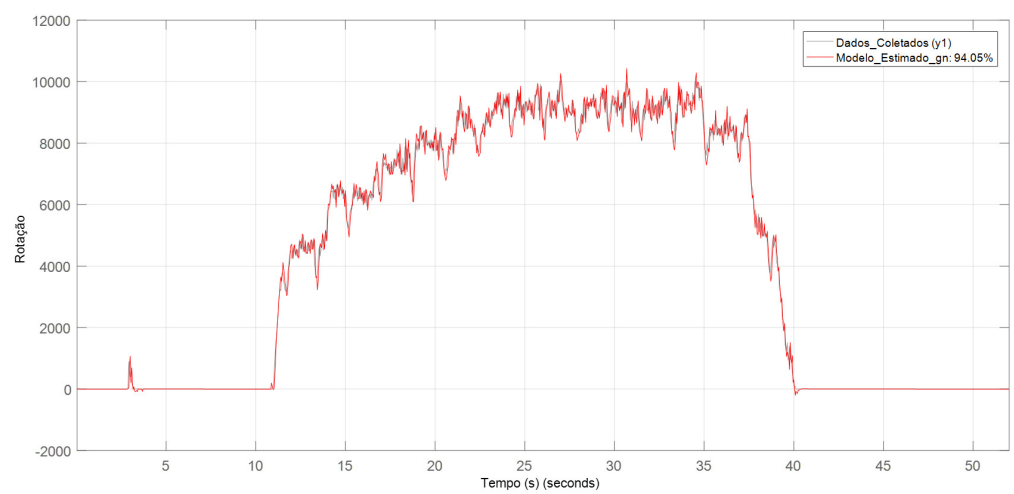

Figura 3: Gráfico do modelo estimado versus dados da plataforma (método de GaussNewton). 
O modelo identificado obteve um ajuste de $94,05 \%$ para o método de gauss-newton e 93,37\% para o método de levenberg-marquardt aos dados da plataforma, conforme ilustrado nas Figuras 3 e 4, onde são apresentados o desempenho comportamental do sistema e a resposta do modelo obtido, respectivamente.

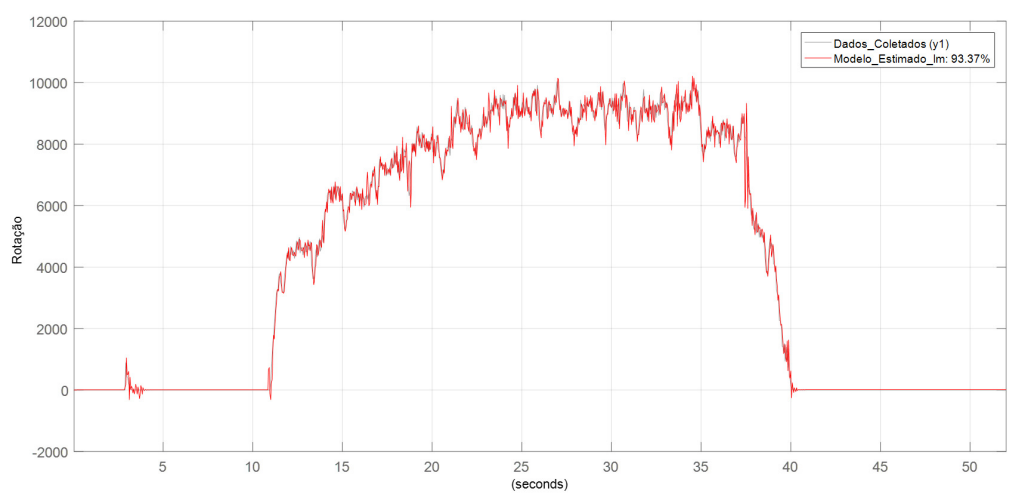

Figura 4: Gráfico do modelo estimado versus dados da plataforma (método de LevenbergMarquadt).

Com a validação cruzada, é possível observar se o modelo é capaz de representar de forma satisfatória os dados experimentais. O resíduo é calculado pela diferença entre os dados observados e os dados calculados pelo modelo, e neste caso, atingiu valores pequenos, oscilando em torno de zero, como se pode observar nas Figuras 5 e 6 . Isto mostra que a diferença entre os dados calculados pelo modelo e os observados não apresentam diferenças significativas.

Porém, para verificar, dentre os métodos, qual tem o menor resíduo e consequentemente quem é o melhor para o problema em questão, é importante fazer a verificação numérica.

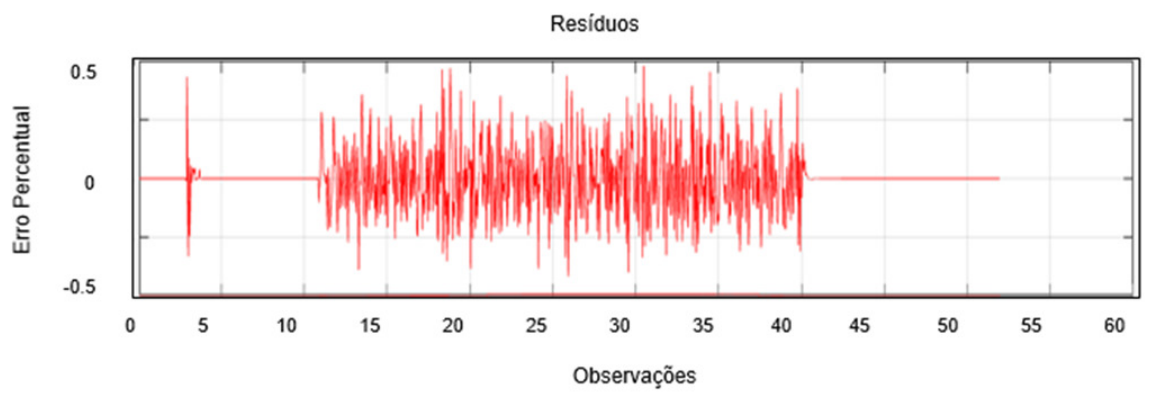

Figura 5: Gráfico do erro pelo método de Gauss-Newton.

Para isso, é calculado oíndice estatístico raiz do erro quadrático médio (RMSE) para cada um dos métodos e observa-se 0,37 para o método de Gauss-Newton e 0,07 para o método de Levenberg-Marquardt. Neste contexto, com a validação realizada por meios subjetivos e quantitativos, é possível afirmar que através de todos os métodos, os modelos 
encontrados representam de forma satisfatória a dinâmica do sistema propulsor eletromecânico modelado, porém o que apresenta melhor resultado é o método de LevenbergMarquardt.

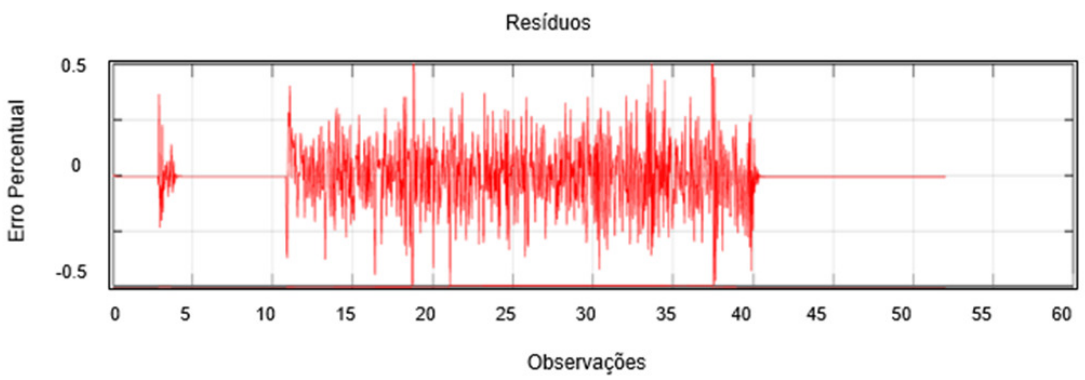

Figura 6: Gráfico do erro pelo método de Levenberg-Marquardt.

\section{Conclusões}

Este artigo trata da formulação de um modelo matemático autorregressivo, por meio da técnica de modelagem caixa preta, tendo como objeto de estudo, a dinâmica comportamental de propulsores eletromecânicos, utilizados em naves não tripuladas do tipo multirrotor. Após a aplicação das etapas da modelagem, obteve-se o modelo $\operatorname{ARMAX}(7,5,4)$, sendo capaz de descrever a dinâmica comportamental dos propulsores eletromecânicos. Estimou-se os parâmetros do modelo pelo método de gauss-newton e método de Levenberg-Marquardt, de onde verificou-se que este último apresentou resultado mais satisfatório por apresentar um erro menor. Os resultados apresentados motivam a investigação de outros métodos (Gauss-Newton adaptado, gradiente de descida, mínimos quadrados não lineares) disponíveis na literatura, a fim de que se possa aprimorar o modelo obtido neste estudo.

\section{Referências}

[1] L. A. Aguirre. Introdução à Identificação de Sistemas: Técnicas Lineares e Não Lineares Aplicadas à Sistemas Reais. UFMG, Belo Horizonte, 2007.

[2] G. E. P. Box, G. M. Jenkins and G. C. Reinsel. Time Series Analysis, Forecasting and Control. Prentice Hall, Englewood Cliffs, 2008.

[3] A. Ost, Modelagem matemática do conjunto ESC-motor-hélice de um VANT utilizando identificação de sistemas, Dissertação de Mestrado, Unijuí, 2015.

[4] L. A. Valer, Modelo matemático ARIMAX de um propulsor eletromecânico utilizado em naves do tipo multirrotor, Dissertação de Mestrado, Unijuí, 2016. 